\title{
Correction to: Periosteal progenitors contribute to load-induced bone formation in adult mice and require primary cilia to sense mechanical stimulation
}

Emily R. Moore*, Ya Xing Zhu, Han Seul Ryu and Christopher R. Jacobs

\section{Correction}

The original article [1] contained two minor errors affecting the labelling of Fig. 3d and Figs. 6b \& 6c; these errors have now been corrected in the respective figures in the original article.

\section{Received: 19 July 2018 Revised: 8 August 2018}

Accepted: 8 August 2018 Published online: 28 August 2018

\section{Reference}

1. Moore ER, et al. Periosteal progenitors contribute to load-induced bone

formation in adult mice and require primary cilia to sense mechanical stimulation. Stem Cell Res Ther. 2018;9:190. https://doi.org/10.1186/s13287018-0930-1. 